\title{
Web 3.0 Monitoring im Stakeholder-Management
}

Dieser Beitrag zeigt auf, welche Möglichkeiten der Einsatz von Web-3.o-Monitoring-Techniken im Stakeholder-Management bietet. Das Ziel dieses Managements ist es, unternehmerischen Vorhaben zu Akzeptanz und Durchsetzungskraft zu verhelfen, indem Ansprüche an Unternehmensentscheide aktiv in den Managementprozess mit eingebunden werden. Stakeholder-Maps stellen diese Ansprüche visuell dar. Sie greifen einerseits auf nicht öffentliche Inhalte zurück und andererseits auf Inhalte, die öffentlich (zumeist im Web) verfügbar sind. Das semantische Web bietet Möglichkeiten, diese öffentlichen Inhalte nicht nur deskriptiv (was wird argumentiert?) darzustellen, sondern auch Zusammenhänge (z.B. Netzwerke, Kontextualisierungen, Referenzierungen, Gewichtungen) aufzuzeigen. Das vorgestellte Framework kann Grundlage für die öffentlichen Inhalte von Stakeholder-Maps sein.

\section{Inhaltsübersicht}

1 Akzeptanz und Durchsetzungskraft unternehmerischer Vorhaben

2 Stakeholder-Mapping und Web 3.0

2.1 Monitoring im Web 3.0

2.2 Emergente Semantik

2.3 Web-3.0-Monitoring-Framework für das Stakeholder-Management

3 Einsatz des Frameworks im StakeholderManagement

3.1 Stakeholder-Management-Szenario

3.2 Beispiel: Einsatz von Stakeholder-Maps bei einem Mittelständler

3.3 Nutzen und Anwendung

3.4 Interaktion mit Stakeholder-Maps

3.5 Vor- und Nachteile des Frameworks

4 Was uns in Zukunft erwarten wird

5 Literatur

\section{$1 \quad$ Akzeptanz und Durchsetzungskraft unternehmerischer Vorhaben}

Die Durchsetzungskraft unternehmerischer Entscheide hängt maßgeblich davon $a b$, wie stark die von ihnen betroffenen Anspruchsgruppen eingebunden werden [Freeman 1984]. Denn Führungskräfte entscheiden heute in einem wachsenden Netzwerk von Interessen (-gruppen). Diese oft komplexe Gemengelage an Anteilseignern, Kunden, Zulieferern, Regulatoren oder gesellschaftlich-ethischen Organisationen ruft nach der Notwendigkeit, Ansprüche systematisch sichtbar zu machen und sie früh in die Entscheidungsfindung einzubeziehen. Das Management von Ansprüchen gegenüber unternehmerischen Vorhaben nennt sich Stakeholder-Management - eine Unternehmensfunktion, die heute in Vorstandsbüros, den Abteilungen für Unternehmensentwicklung, eigenen Abteilungen für Stakeholder-Management (vor allem in US-amerikanischen Unternehmen) oder den Kommunikationsabteilungen angesiedelt ist [Lintemeier et al. 2013].

Ansprüche an unternehmerische Entscheide sind nicht neu - im Gegenteil. Nicht zuletzt dank sozialer Software haben sich Anspruchsgruppen und ihre Kampagnenfähigkeit allerdings professionalisiert. Stakeholder sind in der Lage, global zu agieren und ihre Ansprüche weltweit öffentlichkeitswirksam geltend zu machen. Angriffe kritischer Stakeholder richten sich nicht mehr nur an Unternehmen selbst, sondern an deren gesamte Lieferkette. Der Gestaltungswille nicht wirtschaftlicher Organisationen und Regulatoren hat zugleich zugenommen und klassische Medien verlieren zunehmend an Bedeutung: Meinungsbildung passiert künftig direkt, dezentral oder über Mikromedien. 
Diese unternehmenspolitische Landkarte führt zu einer Neuorientierung der Unternehmenskommunikation, denn die Professionalisierung von Anspruchsgruppen und die Notwendigkeit, unternehmerische Vorhaben nicht mehr nur zu kommunizieren, sondern ihnen zu Durchsetzungskraft zu verhelfen, stellt sie an einen Scheideweg: Entweder professionalisieren sich bestehende Kommunikationsabteilungen im Umgang mit spezifischen Anspruchsgruppen (wie z.B. den Medien) oder sie leisten einen wertschöpfenden Beitrag zur Unternehmensentwicklung. Dieser Scheideweg lässt sich etwa am Nachhaltigkeitsthema erkennen. Während noch vor einigen Jahren Nachhaltigkeit und unternehmerische Verantwortung fast ausschließlich in den Kommunikations- und Marketingabteilungen behandelt wurden, sind sie heute fest im Griff der Finanzressorts. Die Integration von Anspruchsgruppen ist wertschöpfungsrelevant, denn sie kann die Durchsetzungskraft unternehmerischer Vorhaben erhöhen und beschleunigen.

\section{Stakeholder-Mapping und Web 3.0}

Das Management von Ansprüchen geht über eine reine Informationstätigkeit hinaus. Methodisch geht es vielmehr darum, Dialogformate zu schaffen und Ansprüche sichtbar zu machen, sie zu moderieren und in die Entscheidungsfindung einfließen zu lassen. Unternehmerisch geht es darum, Ansprüche entsprechend in unternehmerische Prozesse wie das Procurement, den Einkauf, die Unternehmensentwicklung, die Forschung und Entwicklung etc. tatsächlich einzubinden.

Um diese Ansprüche überhaupt erst sichtbar zu machen, helfen analytische Tools. Analog $\mathrm{zu}$ anderen Managementfunktionen sind sie die Voraussetzung dafür, überhaupt steuern zu können. Zum Beispiel wird keine Marketingabteilung ohne Marktforschung neue Produkte lancieren oder kein Management eine Fusion ohne Machbarkeitsstudien beschließen.
Eine konkrete Möglichkeit, analytische Inhalte sichtbar zu machen, sind visuelle Darstellungen wie z.B. Cognitive Maps, in denen logische Aspekte visuell aufgearbeitet werden. Als vereinfachte Darstellungen komplexer Realitäten erlauben diese, mehrdimensionale Zusammenhänge aufzuzeigen. In einer der »menschlichen Wahrnehmung " nachempfundenen Erweiterung dieser Maps stellte Kosko bereits Mitte der achtziger Jahre die Möglichkeit strategischer Berichterstattung vor [Kosko 1986]. Seine Fuzzy Cognitive Maps bilden nun auch den Angelpunkt des Frameworks von Portmann und Pedrycz [Portmann \& Pedrycz 2013], das im Folgenden für das Management von Ansprüchen herangezogen wird.

Auch im Stakeholder-Management gibt es eine Vielzahl von analytischen Tools: Eines ist das sogenannte Stakeholder-Mapping, das visuelle Aufzeigen von Ansprüchen und Zusammenhängen. Ein Großteil der in solchen Maps dargestellten Ansprüche ist allerdings gar nicht oder nur schwer öffentlich zugänglich. Entsprechend entstehen solche Maps in der Regel auf der Grundlage von persönlichen Einschätzungen, telefonischen Interviews, Redemanuskripten auf privaten Anlässen usw. Doch ein Teil dieser Ansprüche ist durchaus öffentlich einsehbar: Medienberichte wie Zeitungsartikel oder TV-Beiträge, Beiträge in sozialen Medien, veröffentlichte Videos und Buchbeiträge - schlicht alles, was im Web zugänglich ist und damit für die Erstellung von Stakeholder-Maps verwendet werden kann. Und genau das lässt sich über Web 3.0 Monitoring systematisch erfassen und in Stakeholder-Maps einbinden.

\subsection{Monitoring im Web 3.0}

Mit der weltweiten Verbreitung des Begriffs Web 2.0 Ende der neunziger Jahre zeigte O'Reilly erstmals die Überlagerung des "ersten Web« mit dem neuen, sozialen Web auf. Dabei fand laut Blumauer und Pellegrini keine Verdrängung dieses ersten Web statt, sondern eine Erweiterung dessen simpler Webseiten durch 
soziale Medien(-inhalte) [Blumauer \& Pellegrini 2009] und somit durch die Interaktion zwischen Individuen. Die HTML-Seiten des Web 1.0 konnten nun von jedermann mittels einfach zu bedienender sozialer Software um benutzergenerierte Inhalte erweitert werden.

Gegenwärtig erweitert ein ähnliches Phänomen das Web 2.0 zum Web 3.0. In diesem neuen Web 3.0 ergänzt die Vision von BernersLee, Hendler und Lassila vom semantischen Web die genannten Web-2.o-Ansätze. Ihre Vision lässt sich zusammenfassen als Versuch, all die in menschlicher Sprache ausgedrückten Informationen im Web mit einer eindeutigen Beschreibung ihrer Semantik zu versehen, die auch von Computern "verstanden « oder zumindest verarbeitet werden kann [Berners-Lee et al. 2001]. Diese maschinelle Verwendung der Daten aus dem von Menschen geflochtenen Web der Daten ist nur möglich, wenn Informationssysteme deren Bedeutung zuordnen können. In diesem Sinne entstehen aus Daten erst dann Informationen, wenn die Daten (egal ob vom Menschen oder Computer) verstanden werden.

Das durch diese Kombination entstehende Web beruht auf verlinkten Dokumenten, Daten und Applikationen, die automatisch von allen Benutzern im Verlaufe sozialer Interaktion generiert und für ein computerisiertes Informationssystem verständlich gemacht werden. In diesem Web 3.0 verschmilzt das semantische Web, wo Wissen verknüpft wird, mit dem sozialen Web 2.0, das Menschen miteinander verbindet, um dadurch eine Plattform für kollektive Intelligenz zu bieten - in dem durch Kommunikation und spezifische Handlungen von Individuen in einer sozialen Gemeinschaft intelligente Verhaltensweisen hervorgerufen werden können. Da sich verbundene Informationen mit sozialen Beziehungen überlagern, entsteht aus dem Zusammenspiel von Mensch und Informationssystem eine Art künstliche, kollektive Intelligenz, auf die beispielsweise beim Auffinden relevanter Informationen (d.h. analytischer Inhalte) für das Stakeholder-Mapping zurückgegriffen werden kann.
Aufgrund der rasant steigenden Datenmenge im gegenwärtigen Web stellt das Auffinden analytischer Inhalte Kommunikationsverantwortliche heutzutage immer häufiger vor große Herausforderungen, da Signale nur schwach, Daten schwer zugänglich, Ausgangsinformationen vage oder Zusammenhänge nicht direkt ersichtlich sein können. Im Web 3.0 automatisch entstehende Informationsstrukturen erlauben aber ein systematisches Monitoring nach analytischen Inhalten.

\subsection{Emergente Semantik}

Emergente Semantik nutzt Grundsätze des menschlichen Lernens aus, um Computer eines Webinformationssystems die Bedeutung von Daten in einem semiotischen Sinne beizubringen. Semiotik ist die Lehre vom Wesen, der Entstehung (oder der Semiose) und der Verwendung von Zeichen, die einem Verständnis von Information zugrunde liegt. Genauso wie Suchagenten die Bedeutung (oder eben Semantik) aus charakteristischen Interaktionen ableiten, entnimmt emergente Semantik die Bedeutung von Webdokumenten aus der Art und Weise, wie diese von Benutzern gebraucht werden [Cudré-Mauroux 2009]. Das Verfahren nutzt dazu die menschliche Kommunikation und soziale Interaktion im Web 2.0, um darauf aufbauend z.B. mittels Induktion und Klassifikation automatisch Ontologien aufzubauen. Durch diese Ontologien wird dem Web autonom eine "semantische Dimension « hinzugefügt.

In der Informatik sind Ontologien sprachlich gefasste und formal geordnete Darstellungen von Begrifflichkeiten und der zwischen ihnen bestehenden Beziehungen in einem bestimmten Sachverhalt [Gruber 1993]. Diese werden im semantischen Web und folglich auch im Web 3.0 dazu genutzt, Informationen in digitalisierter und formaler Form zwischen Anwendungsprogrammen und Diensten auszutauschen. Dabei unterscheidet sich allerdings das emergente Ontologieerstellungsverfahren von gängigen Verfahren im semantischen Web, in denen Da- 
ten von Experten codiert werden: In traditionellen Verfahren werden dem Web Ontologien manuell von oben nach unten hinzugefügt, wohingegen bei emergenten Verfahren unter Verwendung von Algorithmen zur Verarbeitung der natürlichen Sprache und maschinellem Lernen Ontologien automatisch von unten nach oben »entstehen«.

In den letzten zehn Jahren erarbeitete und standardisierte das $\mathrm{W}_{3} \mathrm{C}$-Konsortium Ontologiesprachen für das semantische Web. Neben $\mathrm{XML}$ und RDF(S) (Resource Description Framework (Schema)) ist OWL (Web Ontology Language) aufgrund des hohen Abstraktionsgrads für Ontologiemodellierung und -entwicklung am populärsten. Mittels eines vielschichtigen Funktionskonstrukts ermöglicht OWL auf relativ einfache Weise ein formales Beschreiben von Beziehungen einer spezifischen Domäne. Nun gibt es aber eine entscheidende Herausforderung: Im Weballtag kommen Informationsstrukturen nicht nur in $\mathrm{W}_{3} \mathrm{C}$-standardisierter Form vor, weswegen sich Informationen syntaktisch und insbesondere auch semantisch stark unterscheiden können.

Emergente Semantik erlaubt es, diese Herausforderung mittels Evolutionsanalyse dezentraler semantischer Strukturen in verteilten Informationssystemen aufzuheben, denn Emergenz tritt durch die spontane Herausbildung neuer Strukturen des Systems infolge des Zusammenspiels der einzelnen Elemente auf. Eine simple Form von emergenter Semantik bildet sich z.B. aus Folksonomien, in denen Webinhalte unter Verwendung sozialer Software von einzelnen Benutzern in einem sozialen, kollabora- tiven Tagging-Prozess meist ohne feste Regeln verschlagwortet werden. Obwohl einzelne Benutzer die Schlagworte (sog. Tags) nach Belieben vergeben, zeigen sich langfristig Konvergenzen zu Namenskonventionen [Rebstock et al. 2008], da die entsprechende Folksonomie die authentische Sprache und das Wissen der Benutzer widerspiegelt. So entfaltet sich (vergleichbar mit der Entwicklung natürlicher Sprachen) ein Vokabular, das als emergente Semantik bezeichnet werden kann. Diese emergente Semantik lässt sich nun in aller Regel mittels der genannten Sprachen des semantischen Web abbilden. Die Kombination beider Verfahren ist das Erfolgsrezept des Web 3.0, weil sich darin die jeweiligen Vorteile ausnutzen lassen.

\subsection{Web-3.0-Monitoring-Framework für das Stakeholder-Management}

Portmann und Pedrycz beschreiben erste Bestandteile des Web-3.o-Monitoring-Frameworks, das in diesem Beitrag für StakeholderManagement verwendet wird [Portmann \& Pedrycz 2013]. Dieses Framework besteht zunächst aus dem »nformationsaggregationsteil«, dem »Informationsrepräsentationsteil« und dem "Schlussfolgerungsteil« (vgl. Abb. 1).

Im Informationsaggregationsteil werden Daten- und Informationsstrukturen aus dem Web extrahiert. Zum einen wird dabei auf bereits vorhandene Informationsstrukturen des semantischen Web zurückgegriffen: Um topdown erstellte Ontologien (die sich mehr oder weniger stark widersprechen können) zu aggregieren, werden diese semantischen Web-Ontologien mittels Soft-Computing-Methoden auf-

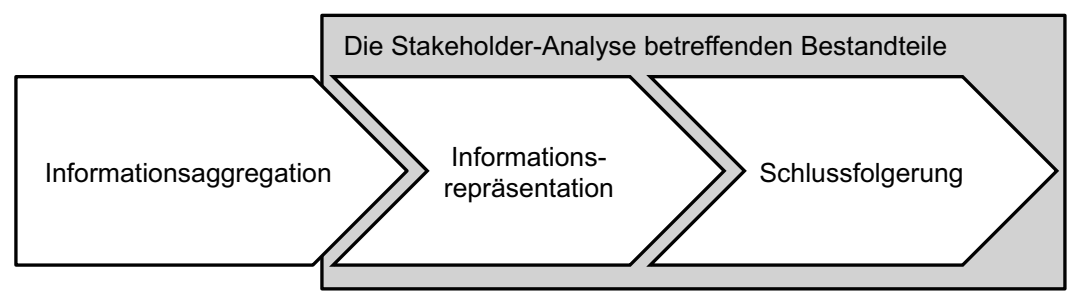

Abb. 1: Web-3.0-Monitoring-Framework im Stakeholder-Management 
einander abgestimmt. Häufig spricht man in diesem Zusammenhang von "Ontologie-Alignement «, womit das Finden von Ähnlichkeiten (sog. Alignements) zwischen Konzepten gemeint ist. Soft-Computing-Methoden verzichten bei so einem Alignement zugunsten qualitativer Charakterisierung und Beschreibung in natürlicher Sprache auf künstlich exakte Werte. Dies erlaubt dem Informationssystem ein anpassungsfähiges und einem menschlichen Ideal nachgebautes, natürliches Verstehen oder Verarbeiten von Semantik.

Im Informationsaggregationsteil können emergente Ontologien auch bottom-up entstehen, wie es Portmann, Kaufmann und Graf beschreiben [Portmann et al. 2012]. Dabei kann die von Benutzern für die Veröffentlichung eines Standpunkts (z.B. in Online-Medienberichten, sozialen Medien, veröffentlichten Videos und Beiträgen) verwendete natürliche Sprache mittels Syntaxanalyse in Komposita zerlegt werden. Mit induktivem Lernen kann ein Computer Zusammenhänge erkennen, die (nach entsprechender Verarbeitung) dem Informationssystem als weitere semantische Dimensionen hinzugefügt werden. Eine mögliche Verarbeitung sieht dabei vor, die mittels induktiven Lernens erkannten Zusammenhänge (wie z.B. Text- und Wortassoziationen) automatisch in lexikalische Relationen zu zerlegen. Auch hier lassen sich durch Soft-Computing-Methoden erkannte Terme automatisch unscharf im lexikalischen Relationskontinuum zuordnen. Dieses Relationskontinuum erstreckt sich von bedeutungsgleichen (Identitäten) über bedeutungsähnliche (Synonyme, Homonyme und Hyperonyme) bis hin zu bedeutungsgegensätzlichen Termen (Antonyme). Im Verlaufe der Bearbeitung können dabei zudem unvergleichbare Terme ausgeschieden werden.

Das Gesetz der großen Zahlen nutzend erlaubt das Verarbeiten der Terme, intellektuelle Funktionen zu imitieren. Weil die Bedeutung der Terme zweideutig sein kann, muss mit Ungenauigkeit und Unsicherheit umgegangen werden können. Eine Möglichkeit, dies zu lösen, ist wieder die Verwendung von Soft-Computing-Methoden. Durch Sammeln von Termen, die miteinander zusammen vorkommen, wird es möglich, Beziehungen zwischen diesen $a b$ zuleiten. Diese Beziehungen (z.B. in der Form Subjekt, Prädikat und Objekte) können als konzeptionelle Informationsstrukturen aufgeschlüsselt werden. Ein großer Vorteil dabei ist, dass, kombiniert mit anderen Merkmalen aus einem Korpus (d.h. aus der Anzahl von gefundenen Webinhalten) und der richtigen Formel (vgl. z. B. [Portmann et al. 2012]), so entdeckte Beziehungen eben als Text- und Wortassoziationsketten gebildet werden können. Eine gezielte Exploration dieser Ketten kann zur Entdeckung neuer Sachzusammenhänge führen. Gerichtete Graphen eignen sich dabei laut Rebstock et al. besonders gut als verwaltende Repräsentationsform [Rebstock et al. 2008].

Sich an Fuzzy Cognitive Maps orientierend, erlaubt der Informationsrepräsentationsteil, die gesammelten Ontologien als Eigenschaftsgraphen visuell darzustellen. Um solche Graphen, die enorme Größenmaße annehmen können (vgl. z.B. [Robinson et al. 2013]), speichern und handhaben zu können, werden Graphdatenbanken eingesetzt. Diese zu traditionellen $\mathrm{Da}$ tenbanken ergänzenden Speichermöglichkeiten erlauben es (z. B. im nachfolgenden Schlussfolgerungsteil), Graphen zu traversieren, um Pfade zu berechnen oder Hotspots zu identifizieren - also Zusammenhänge und Netzwerke aufzuzeigen.

Der Schlussfolgerungsteil von Portmann und Pedrycz erlaubt abschließend logische Folgerungen auf Basis der in der Graphdatenbank gespeicherten Ontologien [Portmann \& Pedrycz 2013]. Neben einer interaktiven Visualisierung der gesammelten analytischen Inhalte als Stakeholder-Maps können diese automatisierten Folgerungen zudem Kommunikationsverantwortliche im Web-Monitoring-Unterfangen unterstützen. Von weniger formalisierten Strukturen grenzen sich ontologiebasierte Graphen da- 
durch ab, dass aus dem in diesen Graphen gespeicherten Wissen logische Schlüsse gezogen werden können. Da Ontologien aus einer Menge von Regeln bestehen, können sie mit Inferenzmaschinen bearbeitet werden. Auf diese Weise kann aus vorhandenem Wissen neues hergeleitet werden [Rebstock et al. 2008].

\section{Einsatz des Frameworks im Stakeholder-Management}

Im folgenden Abschnitt übertragen wir das Web-3.0-Monitoring-Framework auf das Stakeholder-Mapping und zeigen fallstudienartig auf, wie die aufgeführten Tools und Methoden im Stakeholder-Management zum Einsatz kommen können.

\subsection{Stakeholder-Management-Szenario}

Stakeholder-Management in Unternehmen erfolgt grundsätzlich in drei Schritten: erstens der "Stakeholder-Analyse", zweitens dem "Stakeholder-Management-Prozess" und zu guter Letzt den "Stakeholder-Dialogen" (vgl. Abb. 2).

In der Stakeholder-Analyse werden die Ansprüche von Einzelpersonen (nie von Gruppen) zusammengetragen und visuell dargestellt. Unterschieden wird zwischen der Relevanz (z.B. von 1 bis 5) und der Einstellung (z.B. positiv, negativ, neutral). Teil dieser Analyse sind auch sogenannte Stakeholder-Profile, in denen die inhaltliche Position der Person festgehalten ist, ebenso wie Publikationen, Netzwerke sowie die Argumentationslinie für vorgesehene Dialoge. Im Stakeholder-Management-Prozess hingegen steht das Aufsetzen eines Management- prozesses im Vordergrund. Leitende Fragen sind dabei: Wie lassen sich die Stakeholder als Meinungsbildner einsetzen, welche sind wertvolle Ideengeber, welche können unternehmerische Vorhaben torpedieren, welche Allianzenbildung hat Sinn etc. Im letzten Schritt, den sogenannten Stakeholder-Dialogen, werden dann Verhandlungen geführt - von persönlichen Gesprächen bis hin zu mehrtätigen, sehr interaktiven gemeinsamen Dialogformaten. Die Ergebnisse aus den Dialogen fließen laufend in den Management- oder Entscheidungsprozess mit ein.

Das vorgestellte Web-3.0-MonitoringFramework unterstützt den Analyseteil, indem die zusammengetragenen Webinhalte Teil von Stakeholder-Maps werden. Auf den Maps sind personenbezogene Zusammenhänge und Inhalte von Meinungsführern (z.B. Politiker, Journalisten, Leiter von Bürgerinitiativen, Projektleiter bei Nichtregierungsorganisationen (NGOs) u.v. m.) dargestellt (vgl. Abb. 3). Auf den sogenannten Stakeholder-Profilen, die den Maps hinterlegt sind, lassen sich alle zusammengetragenen Informationen zu den Meinungsführern abrufen (z.B. persönliche Einstellungen, verwendete Argumentarien, eventuell sogar Widersprüche, eigene Netzwerke). Die Ergebnisse aus der Webanalyse liefern so eine völlig neue inhaltliche Qualität und decken ggf. sogar bislang nicht sichtbare Zusammenhänge zwischen den Meinungsführern auf. Dabei hilft die Methode von Portmann, Kaufmann und Graf der automatisierten Wissensfindung [Portmann et al. 2012], die basierend auf Webinhalten und unter Anwendung von maschinellen Lernalgorithmen (also Algorithmen, die neues

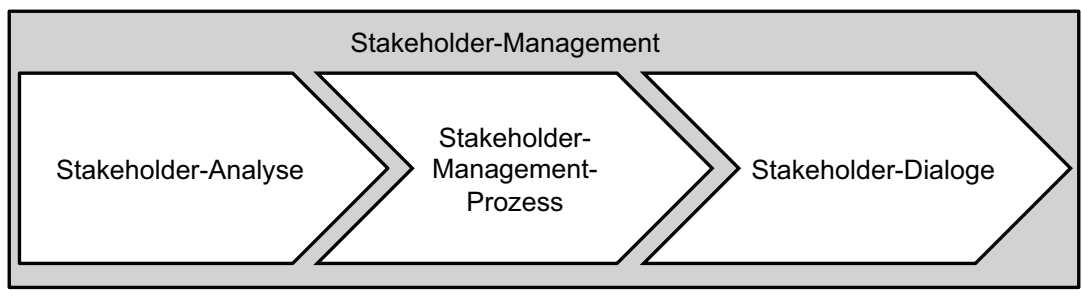

Abb. 2: Unternehmensprozesse des Stakeholder-Management 


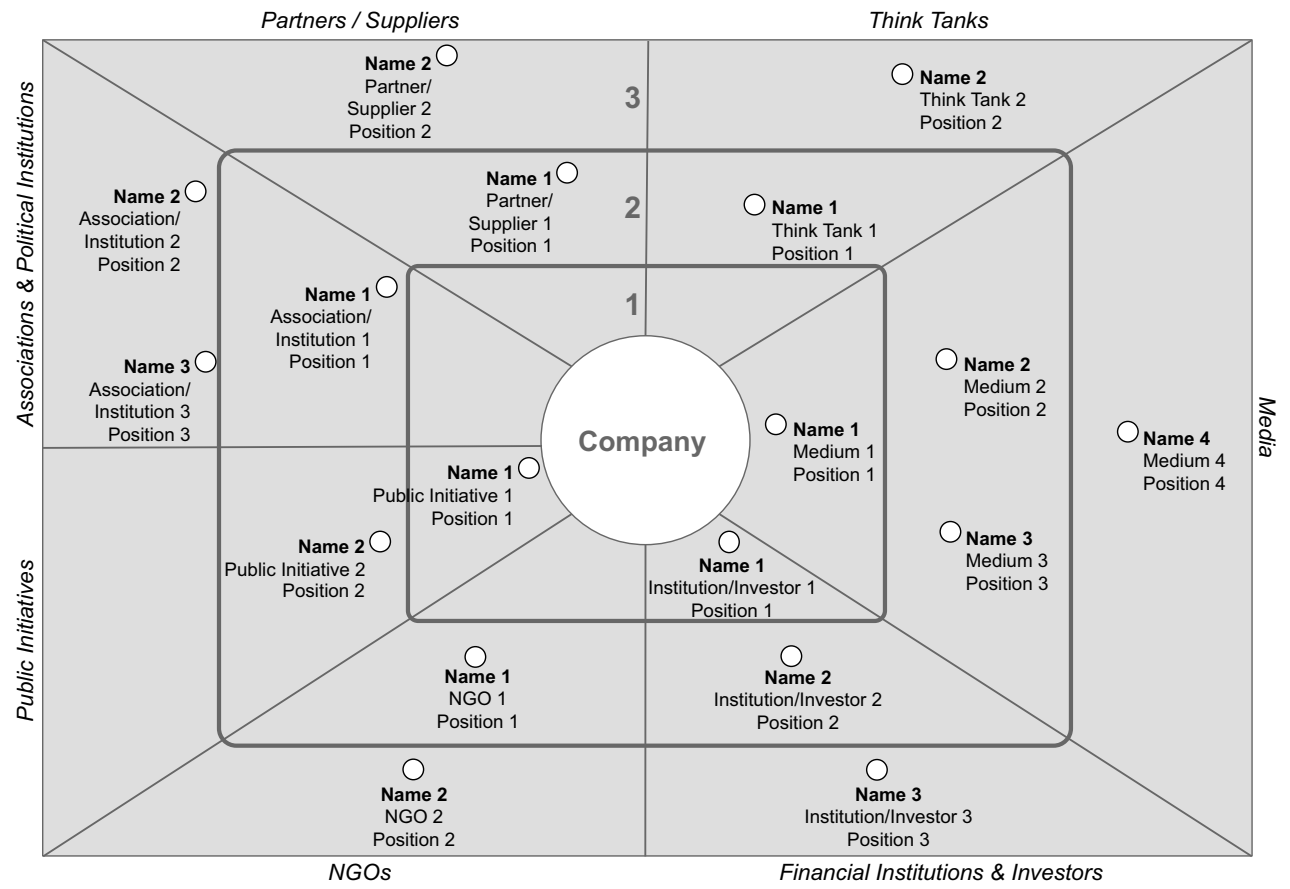

Abb. 3: Beispiel einer Stakeholder-Map

Wissens mittels Induktion herleiten) automatisch aus den gefundenen Inhalten neue semantische Dimensionen erstellt. Diese können in die Stakeholder-Maps einfließen und so auf den ersten Blick für verantwortliche Analysten zuvor unsichtbare Informationszusammenhänge aufzeigen.

\subsection{Beispiel: Einsatz von Stakeholder-Maps bei einem Mittelständler}

Als Beispiel für den Einsatz von StakeholderMaps soll uns ein Schweizer Mittelständler dienen, der global nachhaltige Produkte vertreibt. Die Schwierigkeit dieses Unternehmens ist, dass innerhalb der Branche seine Wettbewerber immer wieder in die Kritik von NGOs geraten, mit dem Vorwurf, sie halten in ihren jeweiligen Produktionsländern Nachhaltigkeitsstandards nicht oder nur ungenügend ein. Die Kritik ist oft undifferenziert und richtet sich pauschal an die gesamte Branche. Unser Beispielunter- nehmen hat es entsprechend schwer, sich vom Wettbewerb durch seine nachhaltigen Produktionsmethoden zu differenzieren.

Eine Konsequenz daraus ist, dass Unternehmensentscheide ständig in der Kritik der NGOs stehen, obschon die Entscheide sogar mit ihren Zielen vereinbar bzw. identisch sind. Die Akzeptanz und Durchsetzungskraft der unternehmerischen Entscheide ist also stark abhängig davon, wie gut die verschiedenen, vor allem kritischen Stakeholder in die Entscheidungsprozesse (z.B. Investitionen in den Produktionsländern) eingebunden sind.

Das Unternehmen hat begonnen, seinen Managementprozess entlang der StakeholderPerspektive neu auszurichten, und bindet dafür Stakeholder heute frühzeitig in Entscheidungen mit ein. Das Spektrum der Einbindung reicht dabei von reinem Informationsaustausch bis hin zu starker Kollaboration (z.B. Entwickeln gemeinsamer Standards). 
Ein entscheidender Teil des neu aufgesetzten Stakeholder-Managements ist die Stakeholder-Analyse (vgl. Abschnitt 3.1). Sie hat das Ziel, alle relevanten Meinungsführer für bestimmte Managementthemen zu identifizieren, sie sichtbar zu machen, die verschiedenen Argumente aufzuzeigen und zu verstehen, um sie dann langfristig in einen Dialogprozess einbinden zu können. Für so eine Analyse kommen verschiedene Stakeholder-Maps zum Einsatz. Auf solchen Maps werden alle Meinungsführer (gruppiert nach Stakeholder-Gruppe, wie z.B. Politiker, kritische NGOs, lokale Behörden, Thinktanks) visuell und inhaltlich aufgetragen. In unserem in Abbildung 3 aufgezeigten Beispiel geht es um die Themen Produktionsstandorte und Beschaffung von Mitteln in Südamerika.

Der kritische Punkt einer solchen Analyse ist die Qualität der Daten. Um ein möglichst vollständiges Bild aller relevanten Meinungsführer zu bekommen, kann das vorgestellte Web-3.0Monitoring-Framework zum Einsatz kommen. Denn durch Web 3.0 Monitoring können Autoren von allen öffentlich zugänglichen Beiträgen (z.B. Politiker, Journalisten, Blogger) systematisch identifiziert und ihre Argumente inhaltlich zusammengetragen werden. Dabei helfen die generierten Ontologien und das Aufzeigen von Zusammenhängen wie z.B. der Verlauf oder die Zusammenstellung ihrer Argumente. Die Informationen für die zugrunde liegenden Ontologien werden einerseits aus bestehenden semantischen Webinformationsstrukturen wie Sammlungen zu Meinungsführern aggregiert und zum anderen aus Webinhalten wie Blogs, sozialen Netzwerken und/oder persönlichen Webseiten (etwa der genannten Meinungsführer) sowie aus allgemein zugänglichen Informationen in Wikis (etwa über die Meinungsführer) etc. angereichert und durch entsprechende Verarbeitung zu Semantik transformiert und zu guter Letzt mittels Soft-Computing-Methoden aufeinander abgestimmt. Die Stakeholder-Map lässt sich so Schritt für Schritt füllen, mit Meinungsführern, deren Einstellungen gegenüber konkreten Managementthemen, deren Argumentarien, der Relevanz für die Entscheidungen und teilweise sogar deren Verbindungen untereinander. Die (unscharfe) Web-3.o-Analyse lässt sogar bereits eine erste Qualifizierung der Relevanz der identifizierten Meinungsführer zu (in der Map in Abbildung 3 durch die Nähe zum Zentrum dargestellt).

In einem zweiten Schritt werden diese öffentlich zugänglichen Daten dann durch die nicht öffentlichen (und entsprechend durch eine Web-3.0-Analyse nicht erfassbaren) Daten angereichert (z.B. persönliche Netzwerke von Journalisten, Meinungsführer, die keine Artikel, Videos oder Zitate publiziert haben bzw. über die nicht berichterstattet wird, Reden in geschlossenen Kreisen, wie Verwaltungsratssitzungen, oder Vorstandsprotokolle, Entscheidungsstrukturen aufseiten der NGOs u.v.m.). Eine Stakeholder-Map ausschließlich aufgrund öffentlicher Daten zu erstellen hat keinen Sinn, da diese einen entscheidenden Teil der Informationen völlig ausblenden würden.

\subsection{Nutzen und Anwendung}

Der Nutzen solcher Maps ist ein analytischer Ausgangspunkt, um zum einen Meinungsführer (auf Personenebene) sichtbar zu machen und zum anderen einen Eindruck der Landschaft der verschiedenen Meinungsführer zu geben. Im Web 3.0 können diese Landkarten erstmalig automatisch mit allem öffentlich Zugänglichen befüllt werden. Auf der Map wird deren Sentiment analysiert und farblich als Einstellung gegenüber dem Unternehmen markiert - z.B. positiv (grün), negativ (rot) oder neutral (weiß). Semantische Webtools können nun den Verantwortlichen auch bei einer Sentimentanalyse unterstützen. Sie ermöglichen z.B. automatisch grammatikalische Beziehungen gesammelter Terme zu analysieren, um Einstellungen von Meinungsführern im Kontext zu erkennen.

Stakeholder-Maps sind "lebendige Dokumente«, die als Grundlage für die Planung von 
Dialogen dienen: Wen muss ich mit welchen Argumenten wie erreichen und in meine Prozesse einbinden bzw. wen muss ich wie untereinander verknüpfen? Wie kann ich einen Meinungsführer für mich gewinnen, indem ich durch wen mit ihm ins Gespräch komme? Diese und weitere Fragen lassen sich durch Stakeholder-Maps vorgängig beantworten, um dann in ein Management der Stakeholder einzufließen.

Der Nutzen des Einsatzes eines Stakeholder-Management-Prozesses für unser Beispielunternehmen war, dass durch die Maps erstmals deutlich wurde, wer überhaupt meinungsführend die Position nachhaltiger Produktion vertritt und wie diese Meinungsführer gegenüber dem Unternehmen eingestellt sind. Dies war die Grundlage für StakeholderProfile, in denen Argumente und Publikationen vollumfänglich zusammengetragen werden konnten. Durch das Nachvollziehen deren Argumente wurden nun mit den Befürwortern nachhaltiger Produktion gemeinsame Studien entwickelt oder sogar Branchenstandards geschaffen. Kritische NGOs konnten bewusst in den Prozess eingebunden werden und sind inzwischen gefragte und willkommene Experten in den Dialogen. Heute unterhalten die Führungsund Fachkräfte der Unternehmung eigenständig Beziehungen zu allen wichtigen Meinungsführern - die allesamt jeweils inhaltlich vorbereitet sind und so konsequent zum Erreichen der unternehmerischen Ziele beitragen. Die Differenzierung gegenüber der Branche wurde erreicht und die Pauschalkritik gegenüber Unternehmensentscheiden ist fast vollständig verebbt.

\subsection{Interaktion mit Stakeholder-Maps}

Das Web-Monitoring-Framework von Portmann und Pedrycz [Portmann \& Pedrycz 2013] erlaubt, wie im vorherigen Beispiel gezeigt, ein interaktives Durchsuchen und Ergänzen von Stakeholder-Maps. Dadurch wird dem Stakeholder-Management ein Tool an die Hand gegeben, mit dem die zunehmende Menge an Webdaten im immer anspruchsvoller werdenden
Monitoring und in der Analyse des StakeholderManagement-Prozesses einfacher bewältigt werden kann. Die Interaktivität erlaubt es den Verantwortlichen nach wie vor, diesen Maps manuell Informationen (d.h. Ansprüche und Zusammenhänge) hinzuzufügen. Diese hinzugefügten Ansprüche und Zusammenhänge stammen dabei aus Quellen, die von den dem Framework zugrunde liegenden Websuchagenten nicht erschlossen werden können.

Wie im vorherigen Abschnitt diskutiert, helfen Suchagenten, emergente Semantik zu etablieren, durch die die Stakeholder-Maps automatisch vom Informationssystem mit analytischen Inhalten befüllt werden: Zum einen aggregieren die Agenten semantische Web-Ontologien und zum anderen konvertieren sie ergänzend natürlichsprachige Webinhalte ebenfalls zu Ontologien. Intelligente, dem menschlichen Lernen nachempfundene Algorithmen helfen, diese Ontologien zu erstellen und aufeinander $a b$ zustimmen. Eine im Framework angewandte Technik ist die Induktion: Eine Deduktion schließt aus gegebenen Voraussetzungen auf einen speziellen Fall, Induktion geht genau den umgekehrten Weg. Eine weitere Technik des Web-Monitoring-Frameworks ist die Klassifikation - d.h. eine Sammlung von abstrakten Klassen, die sich zu anderen Klassen abgrenzen: Dabei werden die einzelnen Klassen algorithmisch durch die Einteilungen von Objekten anhand bestimmter Merkmale (sog. "Prototypen «) erstellt. Auch die Klassifikation ist neuropsychologischer Erkenntnisse nachempfunden, denn die menschliche Begriffsbildung beruht zu einem großen Teil ebenfalls auf unscharfen Klassifikationsprozessen.

Die daraus resultierenden, für das Informationssystem nun verständlichen Ontologien werden (angelehnt an die vorgestellten Fuzzy Cognitive Maps) für die Speicherung zu Graphen aufbereitet. In der Graphentheorie zeichnen Knoten (oder Ecken) und Kanten (Bögen) Ontologien aus [Robinson et al. 2013]. Diese Ontologien werden nun vom Framework zur Spei- 
cherung der semantischen Dimensionen in einer Datenbank herangezogen. Im Gegensatz zu relationalen Datenbanken, die Relationen (Tabellen) und Tupel (Zeilen) verwalten, verwenden Graphdatenbanken Traversionsalgorithmen zur Selektion bestimmter Knoten. Diese können in der Auswertung der Ontologie helfen und so den Kommunikationsverantwortlichen bei Schlussfolgerungen unterstützen.

Die in der Graphdatenbank gespeicherten Ontologien werden herangezogen, um Stakeholder-Maps automatisch mit analytischen Inhalten des Web zu befüllen. Ein Verantwortlicher kann nun im Monitoring- und Analyseprozess die entsprechende Map analysieren (d.h. mittels Browsingfunktion durchsuchen) und (per Insertfunktion) mit zusätzlichen, vertraulichen Ansprüchen und Zusammenhängen anreichern. Der Verantwortliche hat so laufend Zugriff auf inhaltlich valide Profildaten von Stakeholdern auf der Stufe der Meinungsführer, also ganz konkreten Personen. Diese resultierenden Stakeholder-Maps lassen sich als spezielle Graphen sichern und so später wieder (weiter-)bearbeiten. Eine Stakeholder-Analyse kann und sollte nämlich periodisch wiederkehrend durchgeführt werden, um Änderungen der Stakeholder-Attitüden über die Zeit zu erkennen und zu bearbeiten [Lintemeier et al. 2013]. Auf Basis der Stakeholder-Maps kann mit der Schlussfolgerungskomponente auch automatisch gefolgert werden. Auf der Graphentheorie aufbauend, ist die Schlussfolgerungskomponente eine automatisierte Inferenzmaschine, die durch logische Schlussfolgerungsalgorithmen (z.B. durch Traversierung von Pfaden oder Analyse von Hotspots) neue Aussagen aus der bestehenden Ontologie ableiten und so die Kommunikationsverantwortlichen durch interaktive Visualisierung zusätzlich unterstützen kann.

Die Interpretation der Stakeholder-Maps erfolgt dann laufend sowie in Bezug auf Entscheide in Unternehmen. Das heißt, wenn strategische Entscheidungen vorbereitet werden (wie z.B. ein Zukauf von Unternehmen, die Zusam- menlegungen von Business Units, der Wechsel eines Geschäftsmodells, umstrittene Bauvorhaben u.v.m.), wird von Beginn an einbezogen, wen diese Entscheidung betrifft und wie deren inhaltliche Argumentation gegenüber der Entscheidung sein wird. Um Managemententscheidungen durchsetzen zu können, werden die betroffenen Meinungsführer in ein Stakeholder-Management einbezogen - durch Gespräche, Dialoge, gemeinsames Erarbeiten von Teilentscheidungen usw. Stakeholder-Maps und die dahinterliegenden Profile der Stakeholder werden dabei laufend mit Erkenntnissen aus diesem Management aktualisiert. Sie sind damit ein stetes Abbild von Meinungsführern für unternehmerische Entscheide und werden zu einem wichtigen Tool, um Entscheidern gleich von Beginn an zu Akzeptanz und Durchsetzungskraft zu verhelfen.

\subsection{Vor- und Nachteile des Frameworks}

Ein Vorteil des vorgestellten Web-MonitoringFrameworks für das Stakeholder-Mapping ist die Genauigkeit. Das auf neurowissenschaftlichen Erkenntnissen aufbauende Framework (vgl. z.B. [Shi \& Griffiths 2009]) liefert emergente Ontologien [Portmann et al. 2012; Portmann \& Pedrycz 2013], die in Stakeholder-Maps visualisiert zur Verfügung gestellt werden können. Durch die Interaktionsmöglichkeiten (d.h. Browsing- und Insertfunktionen) können Stakeholder-Verantwortliche Informationen aus dem Web durchbrowsen, analysieren und ergänzen (wie z.B. in [Portmann et al. 2012] angedeutet). Die Inferenzmaschine hilft ihnen, neue analytische Inhalte den bestehenden anzufügen.

Ein zweiter Vorteil ist, dass die erstellte Stakeholder-Map mit Anspruchszusammenhängen angereichert werden kann. Dies erlaubt es, nicht nur deskriptiv zu arbeiten, sondern Rückschlüsse auf Meinungsbildung zu ziehen und deutlich gezieltere Stakeholder-Dialoge führen zu können [Freeman 1984; Lintemeier et al. 2013]. Durch eine gezielte Planung dieser Dialoge (z. B. im Managementprozess) können dann Schlüs- 
selthemen identifiziert, Konflikten präventiv vorgegriffen, Interessen vermittelt und auch das Know-how der Stakeholder angezapft werden [Lintemeier et al. 2013]. Innerhalb der Organisation kann zudem ein Verständnis für eine unternehmerische Sozialverantwortung gefördert werden [Freeman 1984]. Darüber hinaus eröffnen Stakeholder-Dialoge oft Chancen für längerfristige Kommunikationsprozesse und -kooperationen mit wichtigen Meinungsführern.

Der wohl größte Nachteil eines (voll-)automatisierten Web-Monitoring- und Analyse-Frameworks kann hingegen sinkende Aufmerksamkeit eines Verantwortlichen sein, z.B. durch übermäßiges Vertrauen in die Emergenz der als Graph gespeicherten Ontologie (und daraus generierten Stakeholder-Maps). Grenzen der semantischen Webtechniken finden sich nämlich überall dort, wo kreative Entscheidungen oder flexible Problemlösungen gefragt sind. Diese Aufgaben kann ein Informationssystem (bislang) nur selten zufriedenstellend lösen, da sie vorwiegend mit gesundem Menschenverstand und persönlichen Präferenzen verbunden sind. Deshalb wird in naher Zukunft wohl kein Informationssystem den Verantwortlichen diese Aufgaben (d.h. selbst den Verstand zu benutzen und automatisch generierte Stakeholder-Maps aktiv zu hinterfragen) vollständig abnehmen können. Zudem ist das Framework nur für öffentliche Daten anwendbar und blendet damit einen wichtigen Teil der notwendigen Informationen aus.

\section{Was uns in Zukunft erwarten wird}

Die technische Abbildung von Ansprüchen und Beziehungen im Stakeholder-Management steckt noch in den Kinderschuhen. Und dennoch, durch die Möglichkeiten des Web 3.0 liegen bereits heute erste Tools und Methoden vor, die weit über die bisherigen Möglichkeiten des Web Monitoring und der Webanalyse hinausgehen. Gleichzeitig entwickelt sich auf Unternehmensseite ein Kommunikationsverständnis, das Abstand nimmt von der reinen In- formationsvermittlung. In unserem Beitrag haben wir deshalb versucht, die Brücke zwischen technologischen und praktischen Möglichkeiten zu schlagen.

Um in Zukunft ausgereifte(re) Frameworks entwickeln zu können, wird abschließend kurz auf HCD (Human-Centered Design) als wichtigste Anforderung eines entsprechenden WebMonitoring-Tools eingegangen. Diese Art des Designs, das darauf abzielt, dass interaktive Tools und Methoden (wie z.B. unser auf Stakeholder-Management adaptiertes Web-Monitoring- und Analyse-Framework) über eine möglichst hohe Usability verfügen, sollte denn auch als Handlungsempfehlung für künftige Implementationen solcher Systeme gesehen werden. Oder wie anders soll beispielsweise die momentan stark zunehmende Stellenflut im Bereich Usability (z.B. bei Facebook, Google, Linkedln) gedeutet werden? In unserem Fall wird eine hohe Usability (und dadurch auch Akzeptanz) im Wesentlichen dadurch erreicht, dass der Stakeholder-Verantwortliche mit seinen Aufgaben, Zielen und Eigenschaften bereits zu Beginn einer Implementation in den Mittelpunkt des Umsetzungsprozesses gestellt wird.

Demzufolge scheint es nichts als folgerichtig, dass beim Bau von (immer stärker) automatisierten Informationssystemen jederzeit der Mensch (in diesem Fall also der StakeholderVerantwortliche) im Mittelpunkt stehen sollte. Nur dieser Verantwortliche kann nämlich "menschliche" (Stakeholder-)Belange bewerten und angemessen einstufen. Dabei wägt er verschiedene, für Informationssysteme alleine nur schwer fassbare Dimensionen (wie z.B. Empathie, ethische Aspekte und Phänomene der realen Welt, metakognitives und implizites Wissen, kollektive Erfahrungen und Traditionen sowie - schlicht und einfach - Know-how) gegeneinander $a b$. Vollautomatisierte Stakeholder-Maps sollten also von ihm hinterfragt und wenn nötig jederzeit auf intuitive Weise angepasst werden können. 
Unsere These ist allerdings, dass mit der (Weiter-)Entwicklung sozialer und semantischer Webtechnologien und der fortschreitenden Professionalisierung von Anspruchsgruppen die Relevanz von analytischen StakeholderManagement-Verfahren weiter steigen wird. Eine wichtige Voraussetzung für die eigene Professionalisierung auf Unternehmensseite sind technische Systeme, die ebenfalls bekannte Denkmuster verlassen und Möglichkeiten bieten, die zunehmend fließende Entscheidungsfindung in Unternehmen zu stützen. Durch das vorgestellte Web-3.0-Monitoring-Framework ist ein erster Schritt in die richtige Richtung getan, auf dem nun ein Stakeholder-Management-Cockpit aufgebaut werden kann, das Kommunikationsverantwortliche bei ihrem (ganzheitlichen) Stakeholder-ManagementProzess unterstützt.

\section{Literatur}

[Berners-Lee et al. 2001] Berners-Lee, T.; Hendler, J.; Lassila, O.: The Semantic Web. Scientific American Magazine 284 (2001), 5, pp. 28-37.

[Blumauer \& Pellegrini 2009] Blumauer, A.; Pellegrini, T.: Social Semantic Web: Web $2.0-$ Was nun? Springer-Verlag, Berlin, Heidelberg, 2009.

[Cudré-Mauroux 2009] Cudré-Mauroux, P.: Emergent Semantics. In: Encyclopedia of Database Systems. Springer-Verlag, Berlin, Heidelberg, 2009, pp. 982-985.

[Freeman 1984] Freeman, R. E.: Strategic Management. A stakeholder approach. Cambridge University Press, Cambridge, 1984.

[Gruber 1993] Gruber, T.: A translation approach to portable ontology specifications. Knowledge acquisition 5 (1993), 2, pp. 199-220.

[Kosko 1986] Kosko, B.: Fuzzy Cognitive Maps. International Journal of Man-Machine Studies 24 (1986), pp. 65-75.

[Lintemeier et al. 2013] Lintemeier, K.; Thiessen, A.; Rademacher, L.: Stakeholder Integration: Zum
Wertschöpfungsbeitrag von Unternehmenskommunikation und Nachhaltigkeitsmanagement. Macromedia, Steinhausen, München, Wien, 2013.

[Portmann \& Pedrycz 2013] Portmann, E.; Pedrycz, W.: Fuzzy Web Knowledge Aggregation, Representation, and Reasoning for Online Privacy and Reputation Management. In: Papapgeorgiou, E. (ed.): Fuzzy Cognitive Maps for Applied Sciences and Engineering: From Fundamentals to Extensions and Learning Algorithms. Springer-Verlag, Berlin, Heidelberg, 2013.

[Portmann et al. 2012] Portmann, E.; Kaufmann, M. A.; Graf, C.: A Distributed, Semiotic-Inductive, and Human-Oriented Approach to Web-Scale Knowledge Retrieval. Proceedings of the 21st ACM International Conference on Information and Knowledge Management, Maui, Hawaii, USA, 2012.

[Rebstock et al. 2008] Rebstock, M.; Fengel, J.; Paulheim, H.: Ontologies-based Business Integration. Springer-Verlag, Berlin, Heidelberg, 2008.

[Robinson et al. 2013] Robinson, l.; Weber, J.; Eifrém, E.: Graph Databases. O'Reilly Media, Sebastopol, 2013.

[Shi \& Griffiths 2009] Shi, L.; Griffiths, T. L.: Neural implementation of hierarchical Bayesian inference by importance sampling. Proceedings of Advances in Neural Information Processing Systems, 2009, pp. 1669-1677.

Prof. Dr. Edy Portmann
Universität Bern
Institut für Wirtschaftsinformatik
Engehaldenstr. 8
CH-3008 Bern
edy.portmann@iwi.unibe.ch
www.iwi.unibe.ch
Dr. Ansgar Thiessen
Knobel Corporate Communications AG
Sennweidstr.35
CH-6312 Steinhausen (ZG)
a.thiessen@knobel.ch
www.knobel.ch

Prof. Dr. Edy Portmann

str.

CH-3008 Bern

edy.portmann@iwi.unibe.ch

Portmann, E.; Thiessen, A.: Web 3.o Monitoring im Stakeholder-Management. HMD - Praxis der Wirtschaftsinformatik 50 (2013), 293, S. 22-33. 